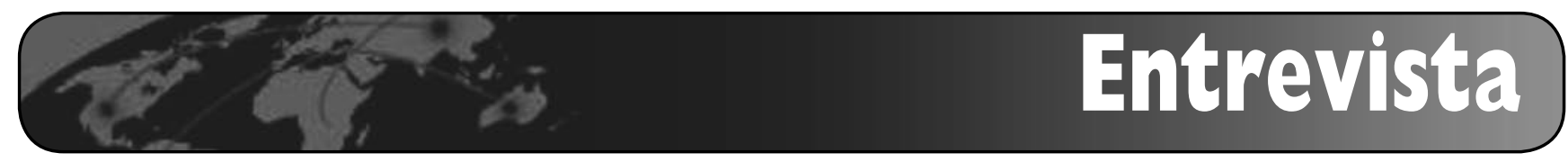

\title{
Situación actual de los estudios cuantitativos de la ciencia. Entrevista con Henk Moed
}

\author{
Por Evaristo Jiménez-Contreras y Daniel Torres-Salinas
}

\begin{abstract}
Jiménez-Contreras, Evaristo; Torres-Salinas, Daniel. "Situación actual de los estudios cuantitativos de la ciencia. Entrevista con Henk Moed”. En: El profesional de la información, 2007, julio-agosto, v. 16, n. 4, pp. 523-526.

DOI: $10.3145 /$ epi.2007.sep.17
\end{abstract}

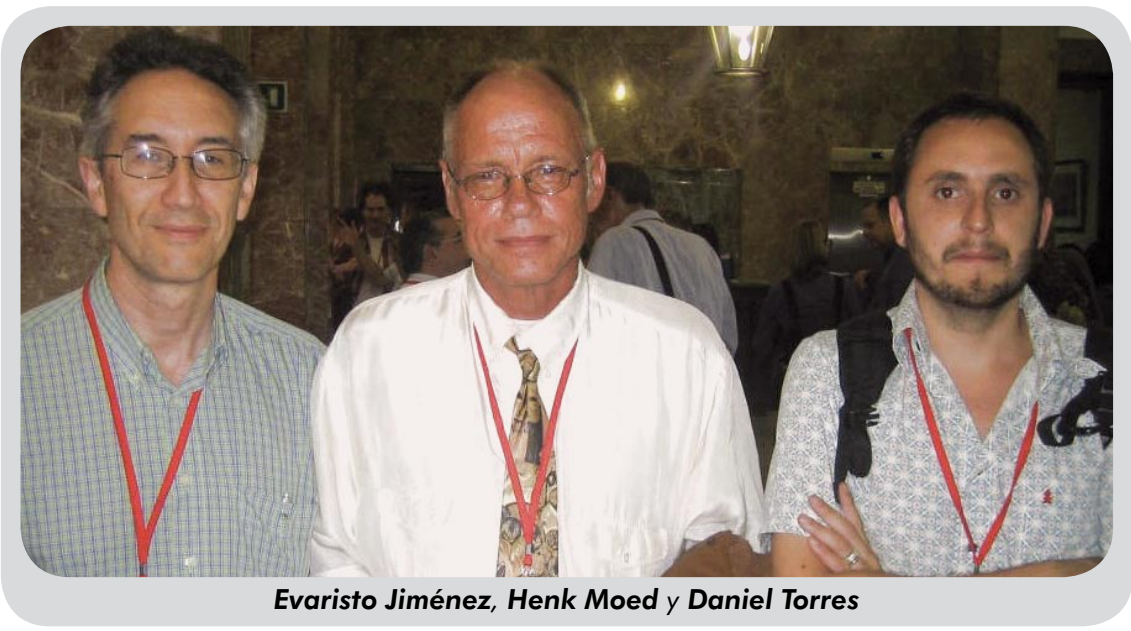

EL PASADO MES DE JUNIO DE 2007, durante los días 25-27, el Campus Serrano del Consejo Superior de Investigaciones Científicas (CSIC) de Madrid acogió la celebración de la $11^{\text {th }}$ International conference of the International Society for Scientometrics and Informetrics ${ }^{1}$.

Este evento, que tiene lugar cada 2 años, es la reunión científica más importante a nivel mundial en el campo de la informetría y la ciencimetría. Este año se celebró por primera vez en España, organizado por el Centro de Información y Documentación Científica (CSIC-Cindoc) con la colaboración como presidente científico de Henk Moed, investigador del Center for Science and Technology Studies $(C W T S)^{2} \mathrm{de}$ la Universidad de Leiden (Holanda). Precisamente para la coordinación científica de los diversos aspectos del congreso, especialmente para la elaboración y edición de sus actas, Henk Moed ha realizado una estancia en España durante los meses de abril y mayo en la Universidad de Granada como profesor invitado del Grupo de Evaluación de la Ciencia y la Comunicación Científica $\left(E C^{3}\right)^{3}$.

Aprovechando su presencia entre nosotros hemos entrevistado a Henk Moed. Creemos que es una ocasión oportuna ya que puede ser una buena piedra de toque para ayudarnos a calibrar el estado actual de los estudios cuantitativos de la ciencia y la tecnología. Como es bien sabido en España es una disciplina con una comunidad científica amplia y bien arraigada en diferentes instituciones. En el ámbito nacional y autonómico es empleada para evaluar los diferentes sistemas de $\mathrm{I}+\mathrm{D}$ en todos sus niveles. Esta circunstancia ha despertado un gran interés por la bibliometría, no solo por parte de los documentalistas, sino también, incluso más, por los propios investigadores que siguen con atención los mecanismos a través de los cuales se evalúa su actividad y se reconoce su trabajo. Buena prueba de este creciente interés se manifiesta en la incorporación y aumento de los contenidos bibliométricos dentro de El profesional de la información. Es por tanto un buen momento para acercar a sus lectores a una de sus figuras más relevantes.

Henk Moed es investigador senior del CWTS que pasa por ser la institución europea más importante e influyente en bibliometría, desde su fundación en 1987. Los trabajos de investigación, propuestas metodológicas e indicadores han sido adoptados por la comunidad científica como verdaderos estándares. Lleva trabajando en dicho centro desde su fundación aunque comenzó a inicios de la década de los ochenta realizando las primeras investigaciones sobre evaluación de instituciones de investigación, especialmente universidades del ámbito de las ciencias y la biomedicina. Sus trabajos se enmarcaron desde el comienzo dentro de la denominada Bibliometría evaluativa donde los indicadores bibliométricos juegan un papel relevante a la hora de la toma de decisiones en la dirección de las políticas científicas. En la década de los noventa la actividad de Moed se ha dirigido hacía 
la obtención de indicadores para evaluar sistemas de I+D nacionales proponiendo diversas medidas estandarizadas que han sido copiadas y empleadas en múltiples trabajos. En este contexto ha dirigido diversos proyectos financiados por la Comisión Europea destinados a detectar la excelencia científica o, más recientemente, a identificar y caracterizar las redes internacionales de colaboración entre universidades. Además es editor asociado de la revista Scientometrics. Su labor científica fue reconocida con la concesión en el año 1999 del Derek John de Solla Price Award, el máximo reconocimiento de la disciplina, que han recibido entre otros investigadores de la talla de Eugene Garfield, Robert K. Merton o Francis Narin. Gran parte de su visión de la bibliometría ha sido recogida en su reciente obra Citation analysis in research evaluation (Moed, 2005) convertida en un clásico desde su publicación.

Su carrera científica abarca casi veinticinco años de dedicación, ¿nos podría explicar como se inició en la bibliometría?

- Después de doctorarme en matemáticas trabajé durante algunos años en la Universidad de Amsterdam en el campo de los estudios sociales de la ciencia, aunque parecía que no había demasiado futuro en ello. En 1981 Anthony van Raan y sus colegas en Leiden obtuvieron un proyecto de investigación del Netherlands Minister of Education con el objetivo de desarrollar indicadores bibliométricos para la política científica de las universidades y yo fui el encargado de realizar el trabajo.

También otros investigadores jugaron un papel muy estimulante para mí. Por ejemplo Cees Le Pair, en aquel momento director asociado de la Foundation for Fundamental Research on Matter (FOM) y Peter Vinker, CEO de Elsevier y fundador de la base de datos Excerpta

\section{Cwts \\ Leiden University}

Medica, ambos amigos personales de Garfield y De Solla Price.

El CWTS goza de un gran prestigio internacional. Haciendo balance de sus dos décadas de existencia, ¿cuáles cree que han sido las principales aportaciones que han realizado? Y, por así decir, ¿dónde reside la clave de su éxito?

- Somos conscientes que los indicadores bibliométricos son herramientas extremadamente útiles y que su desarrollo tiene que realizarse en estrecha colaboración con los agentes evaluados. Siempre hemos procurado estar atentos a posibles errores $\mathrm{y}$, por supuesto, abierto a las posibles críticas y sugerencias que nos pueden ayudar a mejorar. Otro aspecto importante es nuestra concepción de los indicadores bibliométricos como herramientas complementarias de otros métodos como es el caso de la revisión por expertos, esto no significa que la peer review deba ser sustituida por métodos puramente cuantitativos.

Su presencia en España para organizar la $11^{\text {th }}$ International conference of the International Society for Scientometrics and Informetrics le ha permitido entrar en contacto y familiarizarse con el trabajo de diversos grupos de investigación nacionales. ¿Cuál es su opinión sobre el nivel alcanzado por los investigadores españoles?

- Todavía no tengo una visión completa aunque voy haciéndome una idea, pero, efectivamente, existen algunos grupos con un buen nivel que están realizando excelentes contribuciones.

Hablemos ahora del funcionamiento de los mecanismos de evaluación dentro del sistema español de Ciencia y Tecnología. En España es la Cneai (Comisión Nacional de Evaluación de la Actividad Investigadora) la encargada de evaluar y recompensar a sus científicos. Esta institución utiliza como uno de sus principales criterios la valoración positiva de la publicación de trabajos en revistas incluidas en los Journal Citation Reports. ¿Qué opina sobre ese método de evaluación y promoción de los investigadores españoles?

- Bueno, uno siempre debe tener en cuenta la dificultad que entraña evaluar correctamente a los investigadores utilizando únicamente información e indicadores bibliométricos. Actualmente la mayor parte de la investigación científica es producto de un trabajo de grupo por lo que la contribución de un investigador particular no siempre queda reflejada en los indicadores bibliométricos. El sistema empleado en la Cneai tiene su valor y ha funcionado correctamente durante la década de los noventa, sin embargo en la actualidad puede que no sea del todo apropiado. Quizá un nuevo sistema de evaluación en España debería combinar sistemáticamente métodos cuantitativos (bibliometría) con otros cualitativos (peer review).

Uno de los aspectos más controvertidos del sistema evaluador de la Cneai son los criterios empleados en diversas áreas de las ciencias sociales y humanidades que han llevado al diseño de otras herramientas como el In-Recs ${ }^{4}$. ¿Considera la evaluación de las ciencias sociales y especialmente las humanidades como la asignatura pendiente de la bibliometría?, ¿existe alguna alternativa sólida para su evaluación?

- La cobertura de la Web of science es moderada para estas áreas del conocimiento, aunque existen otras alternativas. Es el caso de los índices de citas en ciencias 


\section{Scopus}

sociales y humanidades que se desarrollan en Granada o los índices de las revistas médicas de Valencia. Otra alternativa posible para las humanidades es rastrear la presencia de los libros a través de los opacs (online publics access catalogues) de todo el mundo.

La fuente principal de datos que surte los análisis en que se basan todas las políticas de evaluación no sólo en España, sino en multitud de países, es el conjunto de las bases de datos de Thomson Scientific (antiguo ISI o Institute for Scientific Information). ¿Cuáles cree que son sus principales fortalezas y debilidades?

- El Web of science (conocido antes por Science Citation Index y SCI Search) es una base de datos multidisciplinar. La idea de Eugene Garfield es que el criterio decisivo para incluir una revista como fuente en el índice es el número de veces que citado por revistas que ya están incluidas en el mismo. De esta forma la base de datos tiende a cubrir las mejores revistas en cada campo. Pero hay diferencias entre disciplinas. La cobertura del WoS es excelente en en bioquímica y biología molecular, ciencias relacionadas con la biología humana, medicina clínica, física, astronomía y química, pero es escasa en ciencias sociales y humanidades. En estos últimos campos los libros son fuentes importantes en la comunicación escrita y las barreras lingüísticas juegan su papel.

Respecto al indicador por excelencia, el impact factor (IF), ies posible evaluar la calidad o visibilidad de un país, una institución o un investigador empleando únicamente este indicador o cualquiera de sus derivados?, ¿ha llegado la hora de buscar indicadores más sofisticados?
- En muchos procesos el papel que juega el factor de impacto es excesivamente importante. Debemos tener en cuenta que el $I F$ de los Journal citation reports de Thomson no siempre es calculado de forma exacta y que es susceptible de cierta manipulación. En general este indicador no nos dice demasiado sobre el impacto real que obtiene un trabajo en la ciencia internacional. Toda la comunidad bibliométrica está de acuerdo en que no puede ser utilizado como un sustituto del impacto observado (impacto del artículo concreto, no de toda la revista).

Recientemente en el $B M J^{5}$ se ha despertado el debate sobre el IF por el giro bibliométrico que se va a producir con el Research assesment exercise (RAE). ¿Cómo cree que afectará el empleo de indicadores bibliométricos a la ciencia en el Reino Unido?

- Un estudio bibliométrico longitudinal de la ciencia británica cubriendo casi 20 años reveló en los años anteriores a los $R A E$ tres pautas de comportamiento diferentes que pueden ser interpretadas en términos de la respuesta de los científicos a los criterios principales de evaluación aplicados por los $R A E$. Indicadores bibliométricos sofisticados basados en los impactos observados son, no sólo bastante más informativos del rendimiento de un grupo de investigación, sino también menos fácilmente manipulables que los recuentos brutos de publicación basados en el número de trabajos publicados en revistas con un elevado factor de impacto. Por otro lado, no se puede afirmar que los impactos observados no puedan también ser manipulados por conductas "estratégicas". Evaluadores, evaluados y bibliómetras deben mantenerse alerta ante los efectos imprevistos de su uso.

Otro de los grandes debates en torno a los indicadores se ha generado a partir de la propues-

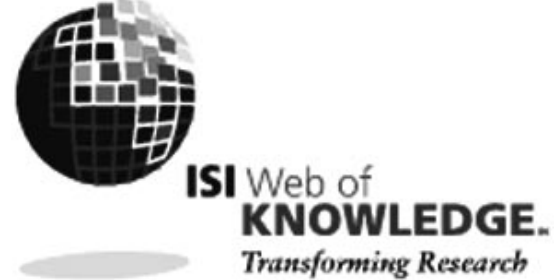

ta del h-index que ha tenido una gran aceptación dentro de la comunidad bibliométrica. ¿Cuáles son sus debilidades y virtudes?, ¿estamos hablando de un indicador definitivo?

- El índice de Hirsch tiene un diseño interesante y creativo pero también presenta graves limitaciones. Por ejemplo no tiene en cuenta las diferencias existentes en las prácticas de publicación y citación de los diversos campos científicos. Otra limitación es que suele favorecer a los investigadores que tienen una carrera investigadora dilatada en el tiempo en perjuicio de aquellos que llevan poco tiempo investigando.

Siguiendo con las alternativas, en los últimos años ha surgido una serie de competidores de las bases de Thomson, principalmente Scopus del grupo Elsevier y Google Scholar. ¿Considera estas iniciativas como competidores serios y maduros de Thomson en la actualidad?

- En el pasado se ha publicado un gran número de estudios sobre los índices de citas de ISI, especialmente en mi propia institución. Hasta el momento no sabemos sobre Scopus todo lo que conocemos sobre las bases de datos de ISI aunque seguramente en el futuro también se publicarán bastantes estudios críticos sobre Scopus. El interesante análisis presentado por Daniel Torres-Salinas en su tesis doctoral es un buen ejemplo. Sin embargo me gustaría apuntar que ISI siempre ha actuado al margen y de forma independiente respecto a los editores científicos mientras que Scopus es un producto del mayor conglomerado editorial, Elsevier, 


\section{Welcome to ISSI 2007 - Madrid!}

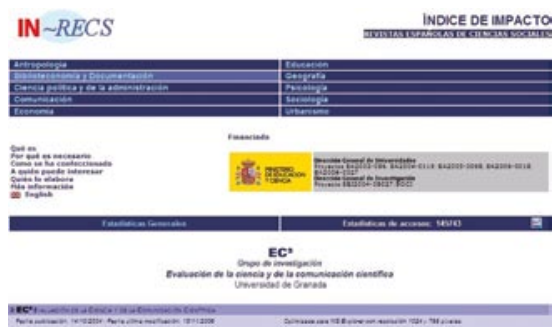

por lo que sus revistas se convierten en la principal fuente de datos de Scopus. Creo que la cobertura de los diferentes campos del conocimiento realizada por Scopus es un tema que merece un examen crítico detenido.

Un tema de gran interés por el cambio de perspectiva que supone en la comunicación de la ciencia es el Open access $(O A)$. En un reciente seminario celebrado en la Universidad de Granada ${ }^{6}$ se presentaron resultados empíricos que evidenciaban que la denominada Open access advantage, por la cual una publicación obtiene mayor citación-impacto por estar en acceso abierto, no se produce en la realidad $i$ No considera su estudio una provocación ante un sistema de publicación que ha levantado enormes expectativas $y$ se ha idealizado en cierta medida entre los profesionales de la información?

- El debate sobre el Open access tiene al menos tres dimensiones: científica, económica y política. Bibliómetras e investigadores del ámbito de la documentación tienen la obligación de asegurar que la información que se emplea en el debate político sea correcta.

Sin duda, el $O A$ supone el nacimiento de un nuevo modelo de comunicación científica, pero sobre todo es una vuelta de tuerca al modelo económico tradicional que se sostenía en las suscripciones. ¿Cuál cree que será la posición de las grandes multinacionales de la edición científica?

- Creo que nadie lo sabe. Muchas de las grandes editoriales cien- tíficas están desarrollando modelos de negocio híbridos que combinan el tradicional principio "los lectores pagan" con el modelo open access donde "los autores pagan". Es difícil predecir en la actualidad cuál de los dos modelos acabará imponiéndose a 10 años vista. Es importante apuntar que la creación de repositorios instituciones de libre acceso tienen unos costes elevados y creo que este aspecto no suele ser tenido en cuenta y en ocasiones se obvia fácilmente.

Para terminar, recientes estudios han puesto de manifiesto la tendencia positiva, en su impacto y en el número de publicaciones, de la ciencia española. Sin embargo usted sostiene que este fenómeno se está produciendo simultáneamente y de forma similar en un gran número de países desarrollados ¿existe una mejora real de la ciencia española o estamos inmersos en una homogeneización en el impacto global que afecta a todos los países, por lo que los resultados de España no son tan espectaculares como creemos?

- Mi impresión es que a nivel macro durante el período 19902006 la producción científica y el impacto observado se han incrementado más rápidamente que en otros países de Europa occidental. Probablemente esto se haya producido por la interacción de tres incentivos de la política científica española: el énfasis puesto en la ciencia, ya que se considera que un sistema de investigación de calidad es crucial para los países desarrollados; la internacionalización de la ciencia española; y, por último, la valoración positiva de la publicaciones recogidas en las revistas de Thomson-ISI. Sin embargo, España está entrando ahora en el grupo de los países que lideran la ciencia mundial por lo que uno debe preguntarse si los objetivos de la política científica deberían ser más diferenciados y personalizados $\mathrm{y}$ cómo se puede desarrollar un sistema de evaluación científica más ajustado. El desafío real quizá deba ser reforzar el rendimiento de la investigación del sistema nacional, distribuyendo los fondos a través de instituciones más dependientes de los criterios de rendimiento/eficacia y al mismo tiempo facilitar y reforzar los controles de calidad y de la gestión de la investigación y de la política científica dentro de las instituciones nacionales de investigación.

$\quad$ Notas
1. http://issi2007.cindoc.csic.es
2. http://cwts.nl
3. http://ec3.ugr.es
4. http://ec3.ugr.es/in-recs/
5. http://www.bmj.com/cgi/content/extract/334/
7593/561
6. http://ec3.ugr.es/seminario/

\section{Bibliografía}

Moed, Henk F. Citation analysis in research evaluation. Dordrecht: Springer, 2005, 346 pp. Isbn 1-4020-3713-9.

Evaristo Jiménez-Contreras; Daniel Torres-Salinas

Grupo EC $C^{3}$ Evaluación de la Ciencia y la Comunicación Científica. Universidad de Granada evaristo@ugr.es torressalinas@gmail.com http://ec3.ugr.es/ 